\title{
Enhancing long-term device stability using thin film blends of small molecule semiconductors and insulating polymers to trap surface-induced polymorphs
}

Tommaso Salzillo, Antonio Campos, Adara Babuji, Raul Santiago, Stefan T. Bromley, Carmen Ocal, Esther Barrena, Rémy Jouclas, Christian Ruzie, Guillaume Schweicher, Yves H. Geerts, Marta Mas-Torrent*

Dr. Tommaso Salzillo, Dr. Antonio Campos, Adara Babuji, Prof. Carmen Ocal, Dr. Esther Barrena, Dr. Marta Mas-Torrent

Institut de Ciència de Materials de Barcelona (ICMAB-CSIC), Campus UAB, 08193

Bellaterra, Spain. E-mail: mmas@icmab.es

Dr. Tommaso Salzillo

Universidad Autònoma de Barcelona, Bellaterra (Cerdanyola del Vallès), 08193, Spain.

Raul Santiago, Prof. Stefan T. Bromley

Departament de Química Física \& Institut de Química Teòrica i Computacional (IQTCUB) Universitat de Barcelona, 08028 Barcelona, (Spain).

Prof. Stefan T. Bromley

Institució Catalana de Recerca i Estudis Avanzats (ICREA), 08010 Barcelona (Spain).

Dr. Rémy Jouclas, Dr.Christian Ruzie, Dr. Guillaume Schweicher, Prof. Yves H. Geerts Laboratoire de Chimie des Polymères, Faculté des Sciences, Université Libre de Bruxelles (ULB), Boulevard du Triomphe 1050 Brussels (Belgium).

Prof. Yves H. Geerts

International Solvay Institutes for Physics and Chemistry, Université Libre de Bruxelles (ULB), Boulevard du Triomphe, 1050 Brussels, Belgium.

Keywords: organic semiconductor, polymorphism, organic field-effect transistor, polymer blend

The lack of long-term stability in thin films of organic semiconductors can often be caused by the low structural stability of metastable phases that are frequently formed upon deposition on a substrate surface. Here, we fabricated thin films of 2,7-dioctyloxy[1]benzothieno[3,2b]benzothiophene $\left(\mathrm{C}_{8} \mathrm{O}-\mathrm{BTBT}-\mathrm{OC} 8\right)$ and blends of this material with polystyrene by solution shearing. Both types of films exhibited the metastable surface-induced herringbone phase (SIP) in all the tested coating conditions. The blended films revealed a higher device performance with a field-effect mobility close to $1 \mathrm{~cm}^{2} / \mathrm{Vs}$, a threshold voltage close to $0 \mathrm{~V}$ and an on/off current ratio above $10^{7}$. In situ lattice phonon Raman microscopy was used to study the stability of the SIP polymorph. It was found that films based on only $\mathrm{C}_{8} \mathrm{O}-\mathrm{BTBT}-\mathrm{OC} 8$ slowly evolved 
to the Bulk cofacial phase, significantly impacting device electrical performance. In contrast, the blended films stabilized the SIP phase, leading to devices that maintained a high performance over 1.5 years. This work demonstrates that blending small-molecule organic semiconductors with insulating binding polymers can trap metastable polymorphs which can lead to devices with both improved performance and long-term stability.

\section{Introduction}

Organic printed electronics is an emerging field for device technology. The active material in such printed devices is an organic semiconductor (OSC), which can be either a conjugated polymer or a small conjugated molecule. Despite the impressive charge carrier mobilities that have been reported for OSCs used in organic field-effect transistors (OFETs) in recent years, two major challenging issues remain unsolved in order to implement these devices in real applications: reproducibility and long-term stability. ${ }^{[1]}$

In thin films of small molecule OSCs the charge transport properties are governed by two parameters: the reorganization energy and the intermolecular electronic couplings. ${ }^{[2]}$ The first parameter is related to the energy loss of a change carrier transfer due to conformational changes and, hence, is mainly related to the molecular structure. On the other hand, the intermolecular electronic couplings between adjacent molecules evaluate the strength of the interactions between the electronic levels of the molecules involved in the charge transfer and is determined by the molecular packing in the solid state. ${ }^{[3,4]}$ Since molecules are bound together through weak van der Waals interactions, they are prone to polymorphism. ${ }^{[5-9]}$ Slight differences between polymorphs can lead to dramatic changes in their mobilities. ${ }^{[10-12]}$ Further, some technologically desirable polymorphs are metastable ${ }^{[13,14]}$ but can degrade with time to the most thermodynamically stable form, thus significantly impacting device performance. Thus, 
controlling the polymorphism of OSCs is of paramount importance to realize high device reproducibility. ${ }^{[15]}$

Among the wide spectrum of studied OSCs, one of the most promising families is based on benzothieno[3,2-b][1]benzothiophene (BTBT), which has led to OFETs with excellent device mobilities. ${ }^{[16,17]}$ The functionalisation of the BTBT core with solubilising groups has been shown to be a promising route to make this material compatible with printing techniques. ${ }^{[18]}$ We focus here on the OSC 2,7-bis(octyloxy)[1]benzothieno[3,2-b]-benzothiophene $(\mathrm{C} 8 \mathrm{O}-$ BTBT-OC, , Figure 1a). ${ }^{[19]}$ It has been reported that the Bulk $\mathrm{C}_{8} \mathrm{O}-\mathrm{BTBT}-\mathrm{OC} 8$ phase crystallizes in a triclinic P-1 system with two molecules per unit cell presenting a displaced cofacial stacking (Figure 1b). ${ }^{[20]}$ A surface-induced phase (SIP) has also been found for this material. ${ }^{[21]}$ whichcrystallizes in a monoclinic P2 $1 /$ c structure with a herringbone packing (Figure 1b). However, it has been observed that spin coated thin films of $\mathrm{C}_{8} \mathrm{O}-\mathrm{BTBT}-\mathrm{OC}_{8}$ exhibiting the SIP phase, slowly convert to the Bulk form over a period of six months. ${ }^{[20]}$ In this work, we fabricated OFETs based on $\mathrm{C}_{8} \mathrm{O}-\mathrm{BTBT}-\mathrm{OC}_{8}$ and blends of this OSC with polystyrene (PS) employing a scalable solution shearing technique. In all cases we could controllably obtain the metastable SIP phase by modifying the film deposition parameters. The Bulk phase on surface could be prepared as single crystals only by drop casting and slow evaporation. Our transport measurements further showed that the SIP phase is more suitable than the Bulk phase for charge transport. Importantly, by blending the OSC with PS, we were able to persistently trap the SIP polymorph. Our work thus demonstrates that using blends of OSCs can be a practical and effective means to achieve long-term device stability with promising metastable polymorphs.

\section{Results and Discussion}

In order to compare the hole transport tendencies in the Bulk and SIP polymorphs of $\mathrm{C}_{8} \mathrm{O}$ BTBT-OC, we first analyzed all independent pairwise HOMO-HOMO (Highest occupied 
molecular orbital) intermolecular electronic couplings ( Номо $_{\text {но }}$ in the two crystal structures using density functional theory (DFT) based calculations (see Figure 1b and Supp.Info.). For the Bulk phase we find a highly anisotropic one dimensional (1D) electronic structure with only

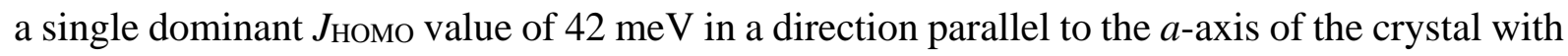
all other $J_{\mathrm{HOMO}}$ values being $\leq 6 \mathrm{meV} \cdot{ }^{[20]}$ In contrast, we find that the SIP polymorphic phase

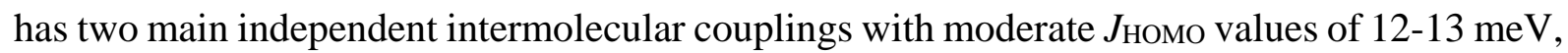
which span over three different directions in the $a b$ plane of the material, indicative of a more 2D electronic isotropy. Thus, although a larger electronic coupling is found in the Bulk phase, the electronic dimensionality in the SIP phase is enhanced, which is also a key aspect to achieve higher device performance. ${ }^{[22]}$

Solutions of $\mathrm{C}_{8} \mathrm{O}-\mathrm{BTBT}-\mathrm{OC}_{8}$ and $\mathrm{C}_{8} \mathrm{O}-\mathrm{BTBT}-\mathrm{OC}_{8}: \mathrm{PS}$ (4:1) in chlorobenzene $2 \%$ wt. were prepared. Two types of PS of different molecular weight were employed, namely, PS3K $\left(\mathrm{M}_{\mathrm{w}}=\right.$ $3000 \mathrm{~g} / \mathrm{mol})$ and PS100K $\left(\mathrm{M}_{\mathrm{w}}=100000 \mathrm{~g} / \mathrm{mol}\right)$. These solutions were deposited by BarAssisted Meniscus Shearing (BAMS) on a $\mathrm{Si} / \mathrm{SiO}_{2}$ substrate with interdigitated gold electrodes at a coating speed of $10 \mathrm{~mm} / \mathrm{s}$ and at a stage temperature of $105^{\circ} \mathrm{C}$ (for details see experimental section). Such a technique has been proven to give rise to homogenous and crystalline large area thin films. ${ }^{[23,24]}$ Interestingly, BAMS is also appealing due to its compatibility with high throughput roll-to-roll processes, of great interest for large-area applications. In these conditions and with the three ink formulations (i.e., $\mathrm{C}_{8} \mathrm{O}-\mathrm{BTBT}-\mathrm{OC}_{8}, \mathrm{C}_{8} \mathrm{O}-\mathrm{BTBT}-\mathrm{OC}_{8}$ :PS3K and $\mathrm{C}_{8} \mathrm{O}-\mathrm{BTBT}-\mathrm{OC} 8 \mathrm{PS} 100 \mathrm{~K}$ ) uniform and crystalline films were obtained (Figure 2a and Figure S1), which were subsequently electrically characterized as active layers in OFETs. Figures 2b-c show representative transfer and output characteristics of the device based on $\mathrm{C}_{8} \mathrm{O}-\mathrm{BTBT}-\mathrm{OC}_{8}: \mathrm{PS} 100 \mathrm{~K}$ (see Figure $\mathbf{S} 2$ for the electrical characteristics of the devices with the other two formulations) elucidating the efficient p-type behavior of the OSC layer. The devices based on only the bare OSC exhibited an average hole mobility $(\mu)$ of $0.09 \mathrm{~cm}^{2} / \mathrm{Vs}$ and a threshold voltage $\left(\mathrm{V}_{\mathrm{TH}}\right)$ of $6.9 \mathrm{~V}$, indicative of some unintentional doping. Remarkably, the 
devices based on blends of PS3K and PS100K revealed a significantly improved performance with average $\mu$ values of 0.43 and $0.9 \mathrm{~cm}^{2} / \mathrm{Vs}$, respectively, and a $\mathrm{V}_{\mathrm{TH}}$ of $-0.9 \mathrm{~V}$ in both cases. In the transfer and output characteristics (Figures 2b-c) some non-idealities commonly found in OFETs can be observed, which can be related to contact effects and charge carrier density mobility dependence. ${ }^{[25-28]}$ The devices based on blends also showed a sharper switch on (small sub-threshold swing), which can be related to a lower density of traps at the OSC/dielectric interface (Table S1). ${ }^{[29,30]}$ Further, the on/off ratio was very high, exceeding $10^{7}$ in the devices based on blends of PS100K. Thus, clearly the blending of the OSC with PS gives rise to higher performing devices with $\mu$ values of up to one order of magnitude higher with respect to the devices based on only the bare OSC.

In order to gain insights into the thin films structural properties, we analysed which polymorphs were present in the prepared solution-sheared films. The two known $\mathrm{C}_{8} \mathrm{O}-\mathrm{BTBT}-\mathrm{OC}_{8}$ polymorphs (i.e., SIP and Bulk) have a completely different crystal packing, nevertheless, when they crystallize on a $\mathrm{Si} / \mathrm{SiO}_{2}$ surface in thin film, they both exhibit a stand-up organization (i.e., the molecules are oriented with their long molecular axis perpendicular to the substrate). In such a case, the use of common out-of-plane X-ray diffraction (XRD) is not enough to distinguish between the two phases because they display an almost identical out-of-plane axis length. ${ }^{[21]}$ Accordingly, the XRD measurements of the films were similar and displayed only the reflections corresponding to the (00l) family, confirming that the $a b$ plane is parallel to the substrate (Figure S3). A spectroscopic study by using mid-infrared and lattice phonon Raman microscopy has been previously reported to be a suitable approach for recognizing the Bulk and SIP phases of $\mathrm{C}_{8} \mathrm{O}-\mathrm{BTBT}-\mathrm{OC}_{8} \cdot{ }^{[19,31]}$ Therefore, the phase of all the freshly fabricated thin films was characterized by Polarization-Modulated Infrared Reflection Absorption Spectroscopy (PM-IRRAS). Thereby, phase differentiation can be achieved as the absorption peak corresponding to the aromatic quadrant stretching is located differently for the SIP phase, 1605 
$\mathrm{cm}^{-1}$, and for the Bulk phase, $1610 \mathrm{~cm}^{-1} .^{[31]}$ The direct comparison of the PM-IRRAS spectra obtained for the our-prepared thin-films with those previously the reported for the SIP and Bulk phases confirmed that, in all the cases, the SIP phase was formed and no traces of the Bulk phase were identified (Figure 2d).

In previous work, we reported that by modifying the substrate temperature and the shearing speed during the thin film preparation, it was possible to tune OSC polymorph formation to produce either a kinetically stable or the thermodynamically stable phase. ${ }^{[15,32]}$ Therefore, in this work the $\mathrm{C}_{8} \mathrm{O}-\mathrm{BTBT}_{-} \mathrm{OC}_{8}$ and $\mathrm{C}_{8} \mathrm{O}-\mathrm{BTBT}-\mathrm{OC}_{8}$ :PS100K ink solutions were deposited at three coating speeds (i.e., $0.1,1,5,10 \mathrm{~mm} \cdot \mathrm{s}^{-1}$ ) and in the temperature range $25-125^{\circ} \mathrm{C}$. It was observed that the domain size, homogeneity and directionality of the crystallites are strongly dependent on the employed deposition parameters (see section 5 in Supporting Info). The most homogeneous films were found at low/intermediate speed when the temperature was low and at high speed when the temperature was high. Thus, the best films were obtained when the deposition speed and temperature led to an instantaneous drying of the solvent. In addition, only some of the thin films prepared at the lowest speed (i.e., $0.1 \mathrm{~mm} \cdot \mathrm{s}^{-1}$ ) showed some preferential alignment of the crystallites.

In Figure S10, the $\mu$ of all the thin films fabricated at different temperatures and speeds are displayed. Generally, we again found that the devices based on blends tend to give rise to better OFET performances. Remarkably, the PM-IRRAS measurements performed on all the thinfilms displayed the same absorption peak corresponding to the SIP phase. Hence, contrary to previously reported works, ${ }^{[15,32]}$ even when a low deposition speed and low temperature were employed to deposit the films by BAMS, the Bulk thermodynamic phase was never formed.

A key issue is to understand the polymorphic stability of the promising metastable SIP phases, since transformation to the thermodynamically favoured Bulk phase would directly impact device performance. Figure 3 (top) shows the polarized optical microscopy (POM) images of 
the thin films based on the bare OSC and blends of the OSC with PS3K and PS100K (deposited at $10 \mathrm{~mm} / \mathrm{s}$ and $105^{\circ} \mathrm{C}$ ) taken on the freshly deposited samples and after 3 months and 1.5 years of storage in atmospheric conditions. Thin films of $\mathrm{C}_{8} \mathrm{O}-\mathrm{BTBT}-\mathrm{OC} 8$ are less smooth than the blended films with smaller crystal domains, and after 3 months the films suffer an significant dewetting effect, as previously reported for similar derivatives (Figure 3). ${ }^{[33-35]}$ After 1.5 years a recrystallization process takes place and clearly defined crystals are observed on the surface which belong to the Bulk phase, as previously observed in spin-coated films of $\mathrm{C}_{8} \mathrm{O}-\mathrm{BTBT}$ $\mathrm{OC}_{8}$ (Figure S11 and S12). ${ }^{[21]}$ In the case of the thin films based on the lower molecular weight PS3K some morphological changes were observed after 3 months, although the films continued to be uniform and continuous (Figure 3). To investigate the nature of these morphology variations at the micrometric scale we analyzed the lattice phonon Raman modes. Lattice phonon Raman spectroscopy has been reported to be a very sensitive tool to study of polymorphism in OSCs ${ }^{[9,36]}$, even for very similar molecular packings. ${ }^{[37]}$ Raman in the low wavenumber region (10-150 $\mathrm{cm}^{-1}$ ) probes the dynamics of the crystal lattice and each structure has its peculiar unambiguous fingerprint. Analyzing two regions of the $\mathrm{C}_{8} \mathrm{O}-\mathrm{BTBT}-\mathrm{OC} 8$ :PS3K films which showed distinct morphologies (i.e., region with unaltered continuous film and areas with defects highlighted with a red circle in the POM image in Figure 3), a different signal in the low Raman shift region was detected, giving the hint that a structural change had occurred. The Raman spectrum of the continuous films coincides with the signal reported by BedoyaMartinez et al. ${ }^{[19]}$ for the SIP phase, whereas the spectrum of the defects are in agreement with the signal of the Bulk phase (Figure 3 (bottom)). It is worth noting that this transformation is very slow and even after 1.5 years no significant conversion in these films is observed. On the other hand, the films with the higher molecular weight PS100K did not show any change in morphology for over 1.5 years and only displayed the SIP phase signal in their Raman spectra. These results importantly indicate that processing an OSC together with a binding polymer 
results not only in an improvement of the OFET performance, but it can enhance the phase stability, especially when higher molecular weight binding polymers are employed.

As measured by AFM, the pristine $\mathrm{C}_{8} \mathrm{O}-\mathrm{BTBT}-\mathrm{OC}_{8}$ films were laterally continuous and smooth at the micrometer scale, exhibiting 2-3 almost complete flat layers of one molecule thickness $(\approx 3 \mathrm{~nm}$ ) on the upper film surface. Further, a quasi-hexagonal molecular order was observed consistent with the herringbone structure of the $a b$ plane of the SIP phase, i.e., with the $c$ axis perpendicular to the surface (see Figure S15 in the Supporting Information). Conversely, in the case of the blended films with PS3K and PS100K, a distinct morphology is observed with some common characteristics, but subtle differences between the two (Figure 4). Firstly, in both cases, the lack of contrast in the frequency shift (insets in Figure $\mathbf{4 b}, \mathbf{4 d}$ ) and lateral force images (see Figure S16 in the Supporting Information), signals extremely sensitive to chemical variations, ${ }^{[34,38]}$ reflects the unique composition of all layer levels exposed at the surface. Secondly, these levels are separated by $\approx 3 \mathrm{~nm}$ (or multiples) high steps that coincides with the molecular length, confirming the standing-up configuration within each layer. Therefore, this is in agreement with the formation of $\mathrm{C}_{8} \mathrm{O}-\mathrm{BTBT}-\mathrm{OC}_{8}$ stacked layers. In fact, high resolution images reveal the same pseudo-hexagonal order of the herringbone packing of the SIP structure at the top-most surface in the blended films as in the pristine $\mathrm{C}_{8} \mathrm{O}-\mathrm{BTBT}-\mathrm{OC}_{8}$ films (Figure 4, Figure S15 and Figure S17 in the Supporting Information). These $\mathrm{C}_{8} \mathrm{O}-\mathrm{BTBT}-\mathrm{OC}_{8}$ molecular layers can be removed in a controlled manner by a layer-by-layer experiment until reaching the PS bottom layer (see Figure 4e-f and below). Hence, these AFM data permit concluding that the upper layers of the two blended systems consist of crystalline $\mathrm{C}_{8} \mathrm{O}-\mathrm{BTBT}-\mathrm{OC}_{8}$ film that covers an underlying PS layer described below.

However, the most striking features differentiating both blends are a large density of dispersed small protrusions (bright spots) in the PS3K blend (Figure 4a, $\mathbf{4 b}$ ) and the presence of several micrometers long stripes, which are more evident in the PS100K (yellow ellipse in Figure 4c and center of Figure 4d). Independently of the PS molecular weight, these stripes have a quite 
regular width and height ( $\sim 150 \mathrm{~nm}$ and $\sim 2.5 \mathrm{~nm}$, respectively) and can be as long as several micrometers. The stripes form local self-organized morphologies connected to a perforated but extended flat region (see lower right part of Figure 4d and Figure S16e). As demonstrated by the layer-by-layer peeling experiment illustrated in the 3D display of Figure 4e (see further details in the Supporting Information), these structures arise from a buried PS-rich layer in contact with the substrate. Note the $3 \mathrm{~nm}$ thickness of the intentionally uncovered layers (Figure 4f). Similarly, buried pellets (indicative of severe layer rupture) lead to faint protrusions that are rarely seen in the PS100K blend but spread over the whole surface in the case of the PS3K blended film. All these observations are a clear evidence of vertical phase separation between $\mathrm{C}_{8} \mathrm{O}-\mathrm{BTBT}-\mathrm{OC}_{8}$ and PS.

The impact of the PS molecular weight on the phase separation and resulting morphology of the PS layer confined at the $\mathrm{SiO}_{2}$ interface (i.e., pellets for the PS3K blend and perforated layer plus stripes for the PS100K case) are in accordance with reports showing that the decrease in PS molecular weight explicitly promote dewetting of PS films on $\mathrm{SiO}_{2} \cdot{ }^{[39]}$ The considerable discontinuity of the buried PS3K layer seems to be at the origin of the more favorable SIP to Bulk phase transformation of $\mathrm{C}_{8} \mathrm{O}-\mathrm{BTBT}-\mathrm{OC}_{8}$ and leads us to suggest that the observed differences on the lateral organization of the polymer at the $\mathrm{SiO}_{2}$ surface influence the stability of the $\mathrm{C}_{8} \mathrm{O}-\mathrm{BTBT}-\mathrm{OC} 8$ :PS films over time.

The electrical stability of the devices stored in ambient conditions was also investigated (Figure 5). The performance of the $\mathrm{C}_{8} \mathrm{O}-\mathrm{BTBT}-\mathrm{OC}_{8}$ films progressively decreased with time. Although this OSC is remarkably electrically stable in environmental conditions, after one year the fieldeffect mobility decreased more than one order of magnitude. Such decrease in performance is accompanied by a large negative shift in the $\mathrm{V}_{\mathrm{TH}}$ of around $6 \mathrm{~V}$. All this is attributed to the structural and morphological changes occurring in the film that results in the accumulation of the OSC material in some areas, leaving behind $\mathrm{SiO}_{2}$ zones free of OSC. This dewetting process 
severely disturbs the conduction percolation path. Consequently, the films did not exhibit fieldeffect behavior any more after 300 days. In order to measure a single crystal of the Bulk phase, we crystallized the material on top of the $\mathrm{Si} / \mathrm{SiO}_{2}$ substrate with a pre-patterned grid of gold contacts by drop casting followed by slow evaporation and confirmed its phase by PM-IRRAS (Figure S13 and S14). Unfortunately, no current was measured, which could be due to nonefficient contacts. Further, the above described anisotropic intermolecular electronic coupling of this polymorph that favours charge transport only along one direction in the plane could also be hindering the transport.

For the blended films the scenario was completely different (Figure 5). Both formulations showed a small enhancement in $\mu$ during the first week after preparation, probably due to some doping or small molecular re-organization. After that, although the device mobility slightly decreased with time, even after 450 days the devices were still working well, with a $\mu$ in the range $0.3-0.6 \mathrm{~cm}^{2} / \mathrm{Vs}$ and with a very low positive $\mathrm{V}_{\mathrm{TH}}$ shift (i.e., around $1 \mathrm{~V}$ ), highlighting the high long-term stability of the $\mathrm{C}_{8} \mathrm{O}-\mathrm{BTBT}-\mathrm{OC}_{8}$ blend for OFETs (Figure S18). Noticeably, the small detected slow conversion from the SIP to the Bulk phase observed in the $\mathrm{C}_{8} \mathrm{O}-\mathrm{BTBT}$ OC8:PS3K films did not seem to affect the electrical performance of the OFETs during this time period.

\section{Conclusions}

In summary, the OSC $\mathrm{C}_{8} \mathrm{O}-\mathrm{BTBT}-\mathrm{OC}_{8}$ was studied as a model system for understanding and controlling polymorphism in devices. This material is known to crystallize in the Bulk phase following a 1D $\pi$-stacking arrangement. However, a metastable SIP phase has also been reported, which shows herringbone structure exhibiting significant 2D intermolecular interactions. Thin films of $\mathrm{C}_{8} \mathrm{O}-\mathrm{BTBT}-\mathrm{OC}_{8}$ and of this material blended with polystyrene of two different molecular weights were prepared by a solution shearing technique and studied as active layers in OFETs. The SIP phase was always formed for the large range of processing 
parameters explored in this work (i.e., varying ink formulation, temperature and coating speed). The devices based on blends exhibited a significantly enhanced performance. Further, the Bulk phase was only formed as large crystals when a solution of the OSC was drop casted on the $\mathrm{Si} / \mathrm{SiO}_{2}$ substrate and let to evaporate very slowly. The best device performances were found for the SIP phase, and blended films exhibited up to one order of magnitude higher field-effect mobility with respect to the devices based on only the bare OSC. More importantly, we demonstrated that while the films based on only the OSC undergo a phase transformation to the Bulk phase in a time scale of months, dramatically hampering the device performance, blending the OSC with PS strongly stabilizes the SIP phase in the films. When the lower molecular weight PS was used, some small local transformation could be detected with no impact in the active layer during the time period studied (i.e., one year and a half). On the other hand, in the films prepared with the higher molecular weight PS no conversion to the Bulk phase was found. The difference in the polymorphic transformation between the two blended films is attributed to a larger discontinuity found in the lower molecular weight PS-rich layer in contact with the $\mathrm{SiO}_{2}$ substrate. Therefore, this work clearly demonstrates that using blended OSCs can not only lead to improved device performance, but can also stabilize metastable polymorphs, of crucial importance to ensure long-term device stability.

\section{Experimental Section}

Device preparation: Bottom gate/bottom contact (BGBC) OFETs were fabricated starting from heavily p-doped $\mathrm{Si} / \mathrm{SiO}_{2}$ wafers from Si-Mat ( $\mathrm{SiO}_{2}$ of thickness $200 \mathrm{~nm}, \mathrm{C}=17.25 \mathrm{nF} / \mathrm{cm}^{2}$ ). Interdigitated gold electrodes (5 nm Cr, $40 \mathrm{~nm} \mathrm{Au}$ ) of various channel lengths ( $\mathrm{L}=50 ; 100$; 150; $200 \mu \mathrm{m}, \mathrm{W} / \mathrm{L}$ ratio kept at 100) were pre-patterned on the $\mathrm{Si} / \mathrm{SiO}_{2}$ substrates by means of photolithography (Micro-Writer ML3 from Durham Magneto Optics Ltd., resolution $5 \mu \mathrm{m}$ ) without requiring the use of a shadow mask. The gold electrodes were treated for 25 minutes by Ozone cleaner and then by immersion for $15 \mathrm{~min}$ in a pentafluorobenzothiol (PFBT, 
purchased from Sigma-Aldrich) solution ( $2 \mu \mathrm{L} / \mathrm{mL}$ in IPA) they were functionalized by creating a self-assembled monolayer (SAM) to reduce the contact resistance.

$\mathrm{C}_{8} \mathrm{O}-\mathrm{BTBT}-\mathrm{OC}_{8}$, synthesized by the procedure previously described, ${ }^{[20]}$ and blends of this semiconductor with atactic polystyrene of molecular weights $3 \mathrm{~K}$ and $100 \mathrm{~K}$ g/mol (SigmaAldrich) in chlorobenzene (CB, Sigma-Aldrich) were deposited with the Bar-assisted meniscus shearing (BAMS) technique. ${ }^{[23]}$ The solution concentration and OSC:polymer ratio were fixed at $2 \% \mathrm{w} / \mathrm{w}$ and $4: 1$, respectively. The substrate temperature and the bar shearing speed were varied from 25 to $125{ }^{\circ} \mathrm{C}$ and from 0.1 to $10 \mathrm{~mm} / \mathrm{s}$, respectively. The final aspects of the fabricated films are displayed in Section 5 of the Supporting Information.

Single crystals of $\mathrm{C}_{8} \mathrm{O}-\mathrm{BTBT}-\mathrm{OC}_{8}$ belonging to the Bulk phase were prepared by drop casting a solution of $\mathrm{C}_{8} \mathrm{O}-\mathrm{BTBT}-\mathrm{OC}_{8} 2 \% \mathrm{w} / \mathrm{w}$ in $\mathrm{CB}$ on a $\mathrm{Si} / \mathrm{SiO}_{2}$ substrate containing pre-patterned gold square contacts (50x50 $\mu \mathrm{m})$. The substrates were covered with a Petri dish and the solvent was left to evaporate overnight.

OFET electrical characterization: The electrical performance was assessed under ambient conditions using an Agilent B1500A semiconductor parameter analyzer coupled with a Karl SÜSS probe station. Transfer characteristics are measured both in the linear and saturation regimes, with $V_{D}$ fixed at $-5 \mathrm{~V}$ and $-20 \mathrm{~V}$ respectively while $\mathrm{V}_{\mathrm{G}}$ is swept between $+10 \mathrm{~V}$ and $20 \mathrm{~V}$ forwards and backwards. Output characteristics are measured under constant $\mathrm{V}_{\mathrm{G}}=+5$, $5,-15$ and $-20 \mathrm{~V}$ while $\mathrm{V}_{\mathrm{D}}$ is swept between $+5 \mathrm{~V}$ and $-20 \mathrm{~V}$, also forward and backwards. The mobility and threshold voltage were extracted for the saturation regime as for the equation (1):

$$
\mu_{S A T}=\frac{2 L}{W C}\left(\frac{\partial \sqrt{\left|I_{D}\right|}}{\partial V_{G}}\right)^{2}
$$

Polarized optical microscopy (POM): Polarized optical microscopy (POM) images were taken with Olympus BX51 microscope equipped with polarizer and analyzer at $90^{\circ}$ in reflection mode. 
Raman: Raman spectra were recorded with a Horiba XploRA PLUS spectrometer equipped with a suitable edge filter and coupled to an Olympus BX51 confocal microscope with 100x and 10x objectives which allow for a lateral resolution lower than $1 \mu \mathrm{m}$ with the higher magnification. This experimental configuration allows gathering information on the polymorphic composition in crystal domains of micrometric dimensions. The excitation wavelength used was the $532 \mathrm{~nm}$ line from a Diode-Pumped Solid State (DPSS) with a nominal power of $25 \mathrm{~mW}$. The power was reduced by neutral density filters to avoid sample damage. The Horiba LabSpec 6 software was used to operate the spectrometer.

PM-IRRAS: Polarization Modulation-Infrared Reflection-Absorption Spectroscopy (PMIRRAS) spectra were recorded by using a Bruker Vertex 70 spectrometer with PM50 module equipped with a liquid-nitrogen-cooled mercury-cadmium-telluride (MCT) photodetector and a permanently aligned Rock Solid interferometer. To reduce absorption due to water and carbon dioxide, the spectrometer was purged by fluxing nitrogen gas. The Bruker spectroscopy software OPUS, version 4.2, was used to operate the spectrometer. Reflection measurements were performed with an aperture of $6 \mathrm{~mm}$ in diameter on the primary side and on aperture of the secondary side at a resolution of $2 \mathrm{~cm}^{-1}$. The incidence angle was set to the Brewster angle of the $\mathrm{SiO}_{2}$ at $57^{\circ}$ to get the best signal-to-noise ratio.

$X$-ray: The thin film orientation was performed by X-ray specular diffractograms in the $2 \theta$ range $5-40^{\circ}$ (step size, $0.02^{\circ}$; time/step, $1 \mathrm{~s} ; 35 \mathrm{~mA}$ x $45 \mathrm{kV}$ ) were collected on a Siemens D5000 diffractometer using as source a Tub ceramic DRX and $\mathrm{Cu}$ anode $(\lambda$ : $\mathrm{CuK} \alpha 1=1.540560$ $\AA, \mathrm{CuK} \alpha 2=1.544390 \AA$ ), equipped with scintillation detector in Bragg-Brentano geometry. The program Mercury2 was used for simulation of X-ray powder patterns on the basis of single crystal data. Thin film orientation and crystal plane were identified by comparison with theoretical morphologies calculated by classic BFDH model (Bravais, Friedel, Donnay and Harker $)^{[40-42]}$ 
AFM: A commercially Atomic Force Microscope from Nanotec Electronica was used to do Atomic force microscopy (AFM). Data were analysed by using the WSxM freeware. ${ }^{[43]}$ We used CrPt-coated Si tips mounted in cantilevers with nominal $\mathrm{k}=3 \mathrm{~N} \cdot \mathrm{m}^{-1}$ (Budget Sensors) for dynamic measurements (topography and frequency shift) and silicon nitride tips mounted on soft cantilevers with $\mathrm{k} \approx 0.01-0.5 \mathrm{~N} \cdot \mathrm{m}^{-1}$ (Veeco) for contact-mode imaging (topography and lateral force) AFM. To provide non-invasive and accurate topographic data, contact mode AFM shown here was collected (unless indicated for induced layer peeling and described in the Supporting Information) at the lowest possible load, i.e., in the attractive regime and as close as possible to the pull off force.

\section{Acknowledgements}

Tommaso Salzillo and Antonio Campos contributed equally to this work. This work was funded by the Spanish Ministry projects (FANCY CTQ2016-80030-R, GENESIS PID2019111682RB-I00， PID2019-110907GB-I00， MICIUN/FEDER RTI2018-095460-B-I00 and MAT2016-77852-C2-1-R (AEI/FEDER, UE)) the Generalitat de Catalunya (2017-SGR13, 2017-SGR-918 and 2017-SGR668) and the Spanish Ministry of Economy and Competitiveness, through the "Severo Ochoa” Programme for Centers of Excellence in R\&D (SEV-2015-0496) and “María de Maeztu” program for Spanish Structures of Excellence (MDM-2017-0767). The authors also thank the European Union’s Horizon 2020 research and innovation programme under the Marie Skłodowska-Curie grant agreement No 811284 (UHMob). We also acknowledge access to supercomputer resources as provided through grants from the Red Española de Supercomputación. T.S. acknowledges the H2020-MSCA-COFUND-2014 Programme (P-SPHERE, Grant agreement 665919). A.B. thanks the Spanish Government financial support through BES-2016-077519 FPI fellowship. This work has been carried out within the framework of the doctoral program $(\mathrm{PhD})$ of Material Science (Department of Physics and Chemistry) of the Universitat Autonoma de Barcelona (UAB). YG is thankful to 
the Belgian National Fund for Scientific Research (FNRS) for financial support through research projects BTBT $n^{\circ}$ 2.4565.11, Phasetrans $n^{\circ}$. T.0058.14, Pi-Fast $n^{\circ}$ T.0072.18, and 2Dto3D n³ 30489208. Financial support from the French Community of Belgian (ARC ${ }^{\circ}$. 20061) is also acknowledged. G.S. acknowledges postdoctoral fellowship support from the FNRS. The authors acknowledge Dr. Gemma Rius and CNM-IMB for the access to the Raman facility.

\section{References}

[1] G. Schweicher, G. Garbay, R. Jouclas, F. Vibert, F. Devaux, Y. H. Geerts, Adv. Mater. 2020, 32, 1905909.

[2] J. L. Brédas, J. P. Calbert, D. A. Da Silva Filho, J. Cornil, Proc. Natl. Acad. Sci. U. S. A. 2002, 99, 5804.

[3] R. Pfattner, S. T. Bromley, C. Rovira, M. Mas-Torrent, Adv. Funct. Mater. 2016, 26, 2256.

[4] S. Riera-Galindo, A. Tamayo, M. Mas-Torrent, ACS Omega 2018, 3, 2329.

[5] A. Brillante, I. Bilotti, R. G. Della Valle, E. Venuti, S. Milita, C. Dionigi, F. Borgatti, A. N. Lazar, F. Biscarini, M. Mas-Torrent, N. S. Oxtoby, N. Crivillers, J. Veciana, C. Rovira, M. Leufgen, G. Schmidt, L. W. Molenkamp, CrystEngComm 2008, 10, 1899.

[6] J. Bernstein, Polymorphism in Molecular Crystals, Oxford University Press, New York, 2002.

[7] D. Gentili, M. Gazzano, M. Melucci, D. Jones, M. Cavallini, Chem. Soc. Rev. 2019, 48, 2502.

[8] R. Pfattner, M. Mas-Torrent, I. Bilotti, A. Brillante, S. Milita, F. Liscio, F. Biscarini, T. Marszalek, J. Ulanski, A. Nosal, M. Gazicki-Lipman, M. Leufgen, G. Schmidt, W. M. Laurens, V. Laukhin, J. Veciana, C. Rovira, Adv. Mater. 2010, 22, 4198.

[9] T. Salzillo, R. G. Della Valle, E. Venuti, A. Brillante, T. Siegrist, M. Masino, F. Mezzadri, A. Girlando, J. Phys. Chem. C 2016, 120, 1831.

[10] V. Coropceanu, J. Cornil, D. A. da Silva Filho, Y. Olivier, R. Silbey, J.-L. Bredas, Top. Curr. Chem. 2007, 107, 926.

[11] M. Mas-Torrent, C. Rovira, Chem. Rev. 2011, 111, 4833.

[12] H. Chung, Y. Diao, J. Mater. Chem. C 2016, 4, 3915.

[13] G. Giri, R. Li, D. M. Smilgies, E. Q. Li, Y. Diao, K. M. Lenn, M. Chiu, D. W. Lin, R. Allen, J. Reinspach, S. C. B. Mannsfeld, S. T. Thoroddsen, P. Clancy, Z. Bao, A. Amassian, Nat. Commun. 2014, 5, 3573.

[14] G. Giri, S. Park, M. Vosgueritchian, M. M. Shulaker, Z. Bao, Adv. Mater. 2014, 26, 487.

[15] A. Tamayo, S. Riera-Galindo, A. O. F. Jones, R. Resel, M. Mas-Torrent, Adv. Mater. Interfaces 2019, 6, 1900950.

[16] H. Ebata, T. Izawa, E. Miyazaki, K. Takimiya, M. Ikeda, H. Kuwabara, T. Yui, J. Am. Chem. Soc. 2007, 129, 15732.

[17] R. Janneck, N. Pilet, S. P. Bommanaboyena, B. Watts, P. Heremans, J. Genoe, C. Rolin, Adv. Mater. 2017, 29, 1703864. 
[18] C. Liu, T. Minari, X. Lu, A. Kumatani, K. Takimiya, K. Tsukagoshi, Adv. Mater. 2011, 23, 523.

[19] N. Bedoya-Martínez, B. Schrode, A. O. F. Jones, T. Salzillo, C. Ruzié, N. Demitri, Y. H. Geerts, E. Venuti, R. G. Della Valle, E. Zojer, R. Resel, J. Phys. Chem. Lett. 2017, 8, 3690.

[20] C. Ruzié, J. Karpinska, A. Laurent, L. Sanguinet, S. Hunter, T. D. Anthopoulos, V. Lemaur, J. Cornil, A. R. Kennedy, O. Fenwick, P. Samorì, G. Schweicher, B. Chattopadhyay, Y. H. Geerts, J. Mater. Chem. C 2016, 4, 4863.

[21] A. O. F. Jones, Y. H. Geerts, J. Karpinska, A. R. Kennedy, R. Resel, C. Röthel, C. Ruzié, O. Werzer, M. Sferrazza, ACS Appl. Mater. Interfaces 2015, 7, 1868.

[22] A. Campos, N. Oxtoby, S. Galindo, R. Pfattner, J. Veciana, S. T. Bromley, C. Rovira, M. Mas-Torrent, CrystEngComm 2016, 18, 6149.

[23] F. G. Del Pozo, S. Fabiano, R. Pfattner, S. Georgakopoulos, S. Galindo, X. Liu, S. Braun, M. Fahlman, J. Veciana, C. Rovira, X. Crispin, M. Berggren, M. Mas-Torrent, Adv. Funct. Mater. 2016, 26, 2379.

[24] I. Temiño, F. G. Del Pozo, M. R. Ajayakumar, S. Galindo, J. Puigdollers, M. MasTorrent, Adv. Mater. Technol. 2016, 1, 1600090.

[25] E. G. Bittle, J. I. Basham, T. N. Jackson, O. D. Jurchescu, D. J. Gundlach, Nat. Commun. 2016, 7, DOI 10.1038/ncomms10908.

[26] H. H. Choi, K. Cho, C. D. Frisbie, H. Sirringhaus, V. Podzorov, Nat. Mater. 2018, 17, 2.

[27] Y. Xu, H. Sun, A. Liu, H. Zhu, B. Li, T. Minari, F. Balestra, G. Ghibaudo, Y. Y. Noh, Adv. Funct. Mater. 2018, 28, 1803907.

[28] M. Krammer, J. W. Borchert, A. Petritz, E. Karner-Petritz, G. Schider, B. Stadlober, H. Klauk, K. Zojer, Crystals 2019, 9, 85.

[29] A. Campos, S. Riera-Galindo, J. Puigdollers, M. Mas-Torrent, ACS Appl. Mater. Interfaces 2018, 10, 15952.

[30] M. H. Yoon, C. Kim, A. Facchetti, T. J. Marks, J. Am. Chem. Soc. 2006, 128, 12851.

[31] B. Schrode, A. O. F. Jones, R. Resel, N. Bedoya, R. Schennach, Y. H. Geerts, C. Ruzié, M. Sferrazza, A. Brillante, T. Salzillo, E. Venuti, ChemPhysChem 2018, 19, 993.

[32] S. Galindo, A. Tamayo, F. Leonardi, M. Mas-Torrent, Adv. Funct. Mater. 2017, 27, 1700526.

[33] Y. Diao, L. Shaw, Z. Bao, S. C. B. Mannsfeld, Energy Environ. Sci. 2014, 7, 2145.

[34] A. Pérez-Rodríguez, I. Temiño, C. Ocal, M. Mas-Torrent, E. Barrena, ACS Appl. Mater. Interfaces 2018, 10, 7296.

[35] A. Babuji, I. Temiño, A. Pérez-Rodríguez, O. Solomeshch, N. Tessler, M. Vila, J. Li, M. Mas-Torrent, C. Ocal, E. Barrena, ACS Appl. Mater. Interfaces 2020, 12, 28416.

[36] A. Brillante, I. Bilotti, R. G. Della Valle, E. Venuti, A. Girlando, CrystEngComm 2008, 10, 937.

[37] T. Salzillo, S. D’Agostino, A. Rivalta, A. Giunchi, A. Brillante, R. G. Della Valle, N. Bedoya-Martínez, E. Zojer, F. Grepioni, E. Venuti, J. Phys. Chem. C 2018, 122, 18422.

[38] W. W. Scott, B. Bhushan, Ultramicroscopy 2003, 97, 151.

[39] S. Lee, W. Lee, N. L. Yamada, K. Tanaka, J. H. Kim, H. Lee, D. Y. Ryu, Macromolecules 2019, 52, 7524.

[40] M. G. Friedel, Bull. Minéralogie 1907, 30, 326.

[41] J. D. H. Donnay, D. Harker, J. Mineral. Soc. Am. 1937, 22, 463.

[42] A. Bravais, Etudes Cristallographiques, Gauthier Villars, Paris, 1866.

[43] I. Horcas, R. Fernández, J. M. Gómez-Rodríguez, J. Colchero, J. Gómez-Herrero, A. M. Baro, Rev. Sci. Instrum. 2007, 78, 013705. 
a)<smiles>CCCCOc1ccc2c(c1)sc1c3ccc(OCCC)cc3sc21</smiles>
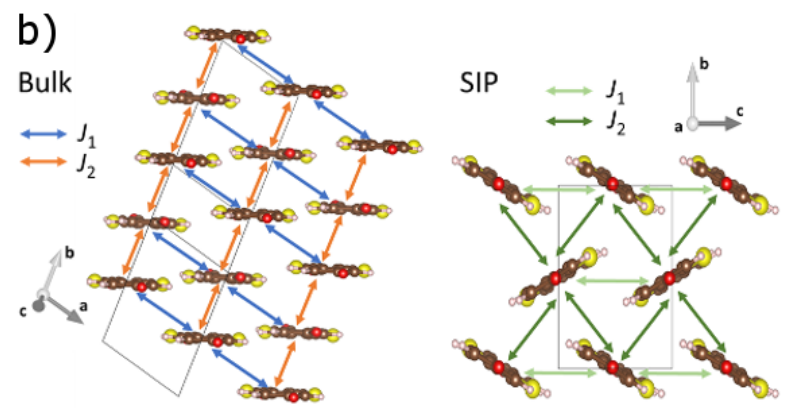

\begin{tabular}{lcc} 
Polymorph & \multicolumn{2}{c}{$J_{\text {номо }}$ (meV) } \\
\hline Bulk & $J_{1}$ & 42 \\
Bulk & $J_{2}$ & 6 \\
Bulk & $J_{3}$ & 4 \\
Bulk & $J_{4}$ & 3 \\
Bulk & $J_{5}$ & 2 \\
\hline SIP & $J_{1}$ & 13 \\
SIP & $J_{2}$ & 12 \\
SIP & $J_{3}$ & $\sim 0$ \\
\hline
\end{tabular}

Figure 1. a) Molecular structure of $\mathrm{C}_{8} \mathrm{O}-\mathrm{BTBT}-\mathrm{OC}_{8}$. b) Crystal structures of the Bulk and SIP polymorphs (the grey solid lines indicate the unit cell boundaries). Coloured arrows indicate directions corresponding to the largest $\mathrm{J}_{\text {номо }}$ values in each case. The rest of the $\mathrm{J}$ values directions are shown in the Supp. Info. Right: list of all calculated independent Jномо values listed in decreasing magnitude for each polymorph. 

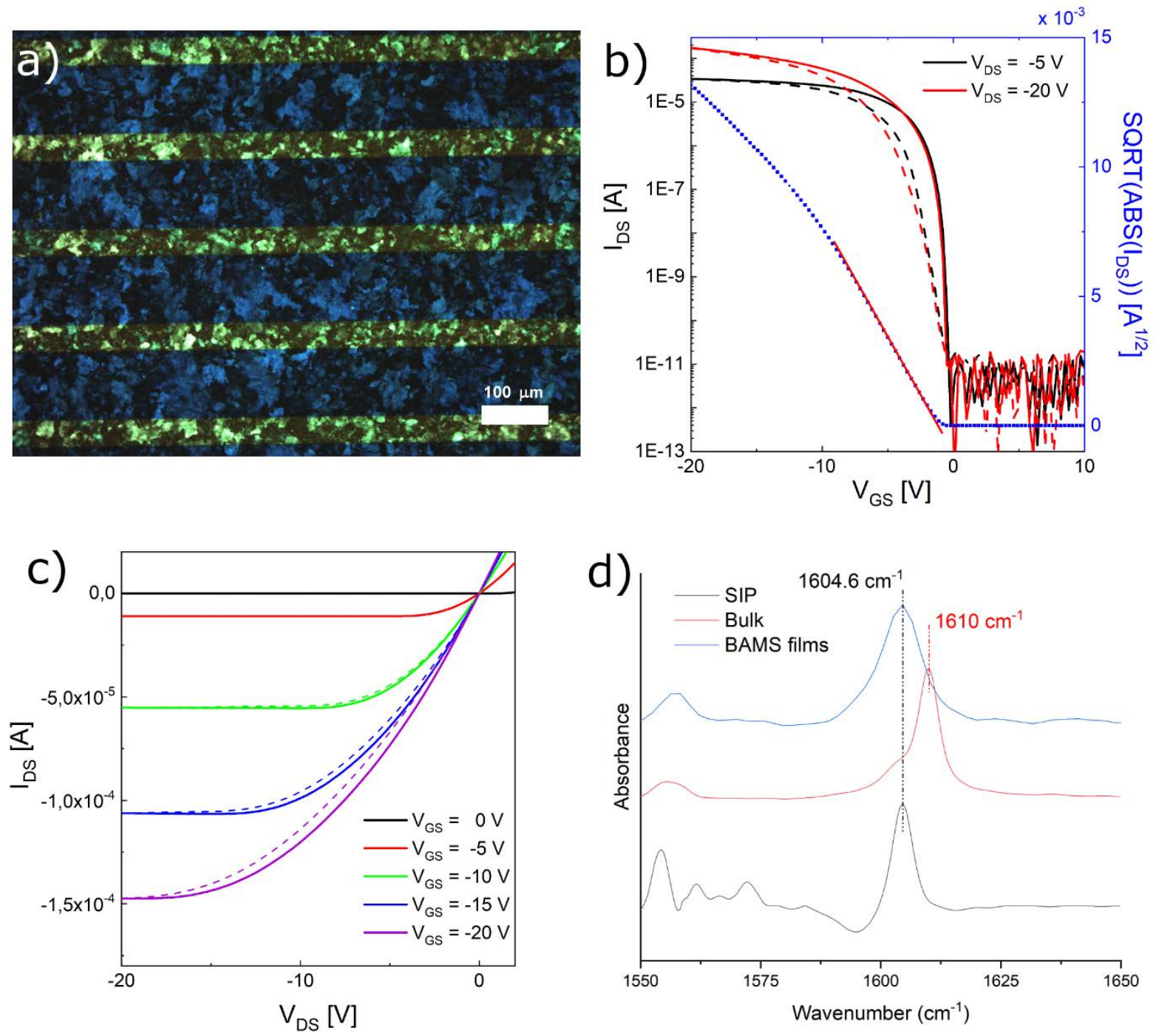

Figure 2. a) Polarised optical microscopy image of a $\mathrm{C}_{8} \mathrm{O}-\mathrm{BTBT}-\mathrm{OC}$ :PS100K thin film prepared at $105{ }^{\circ} \mathrm{C}$ with a shearing speed of $10 \mathrm{~mm} / \mathrm{s}$. Corresponding transfer (dot-lines backward sweep) b) and output c) electrical characteristics of the $\mathrm{C}_{8} \mathrm{O}-\mathrm{BTBT}-\mathrm{OC} 8$ :PS100K thin film prepared $(\mathrm{L}=150 \mu \mathrm{m}, \mathrm{W} / \mathrm{L}=100)$. This device exhibited a saturation mobility of 0.92 $\mathrm{cm}^{2} / \mathrm{Vs}$, with a reliability factor calculated according to ref. 26 of $63 \%$. d) Representative PMIRRAS performed on one of the solution sheared $\mathrm{C}_{8} \mathrm{O}-\mathrm{BTBT}-\mathrm{OC}_{8}$-based film $\left(\mathrm{C}_{8} \mathrm{O}-\mathrm{BTBT}\right.$ $\left.\mathrm{OC}_{8}: \mathrm{PS} 100 \mathrm{~K}\right)$ plotted together with the reference spectra of the SIP and Bulk phase. 

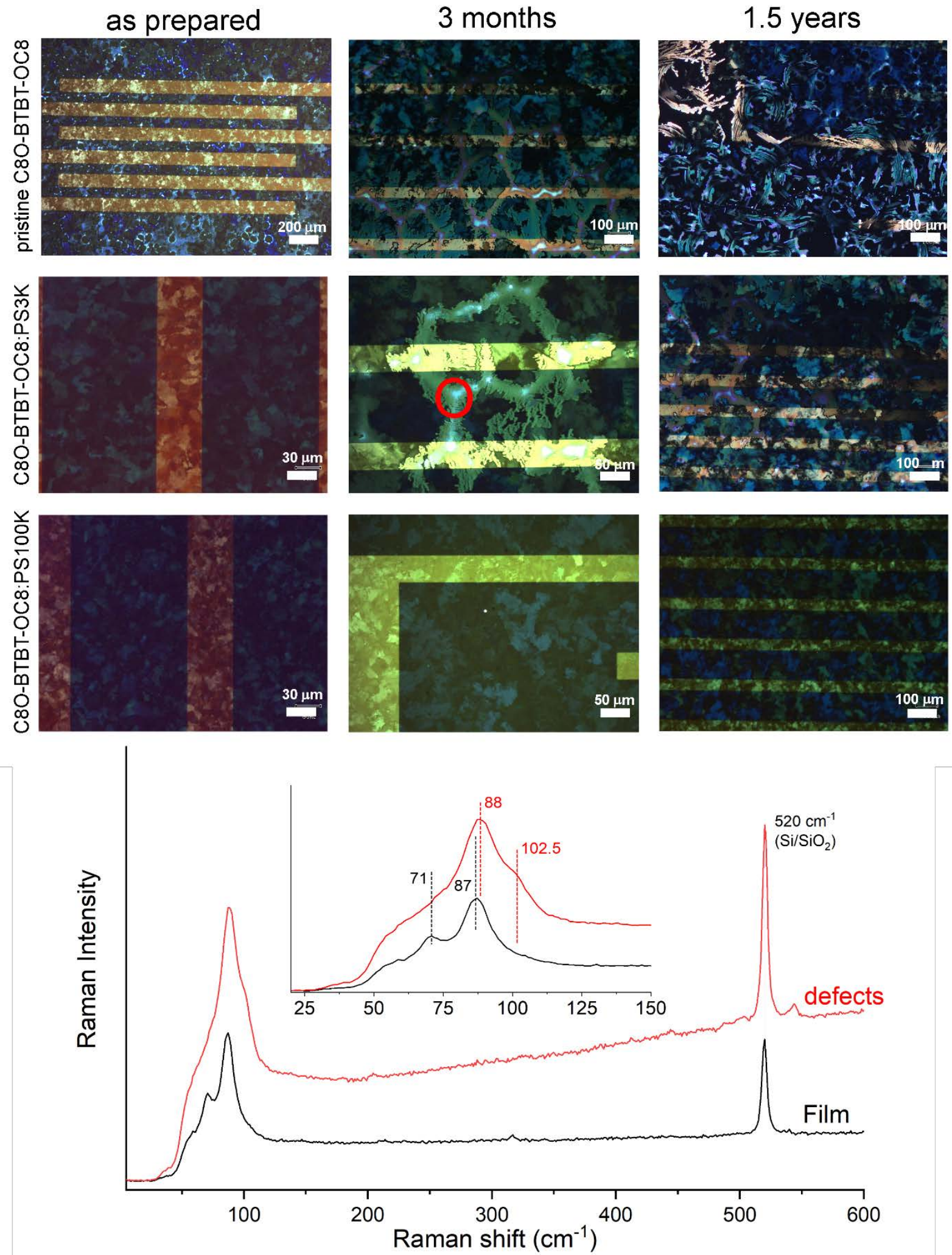

Figure 3. Top. POM images of the OSC thin films based on $\mathrm{C}_{8} \mathrm{O}-\mathrm{BTBT}-\mathrm{OC}_{8}, \mathrm{C}_{8} \mathrm{O}-\mathrm{BTBT}-$ OC8:PS3K and $\mathrm{C}_{8} \mathrm{O}-\mathrm{BTBT}-\mathrm{OC} 8: \mathrm{PS} 100 \mathrm{~K}$ (deposited at $10 \mathrm{~mm} / \mathrm{s}$ and $105^{\circ} \mathrm{C}$ ): freshly prepared, after 3 months and after 1.5 years. Bottom. Raman spectra taken at two different regions of the $\mathrm{C}_{8} \mathrm{O}-\mathrm{BTBT}-\mathrm{OC}_{8}$ :PS3K films three months aged. The red spectrum corresponds to the areas with defects (marked as a red circle in the POM image) and the black one to the areas which have not been affected. 

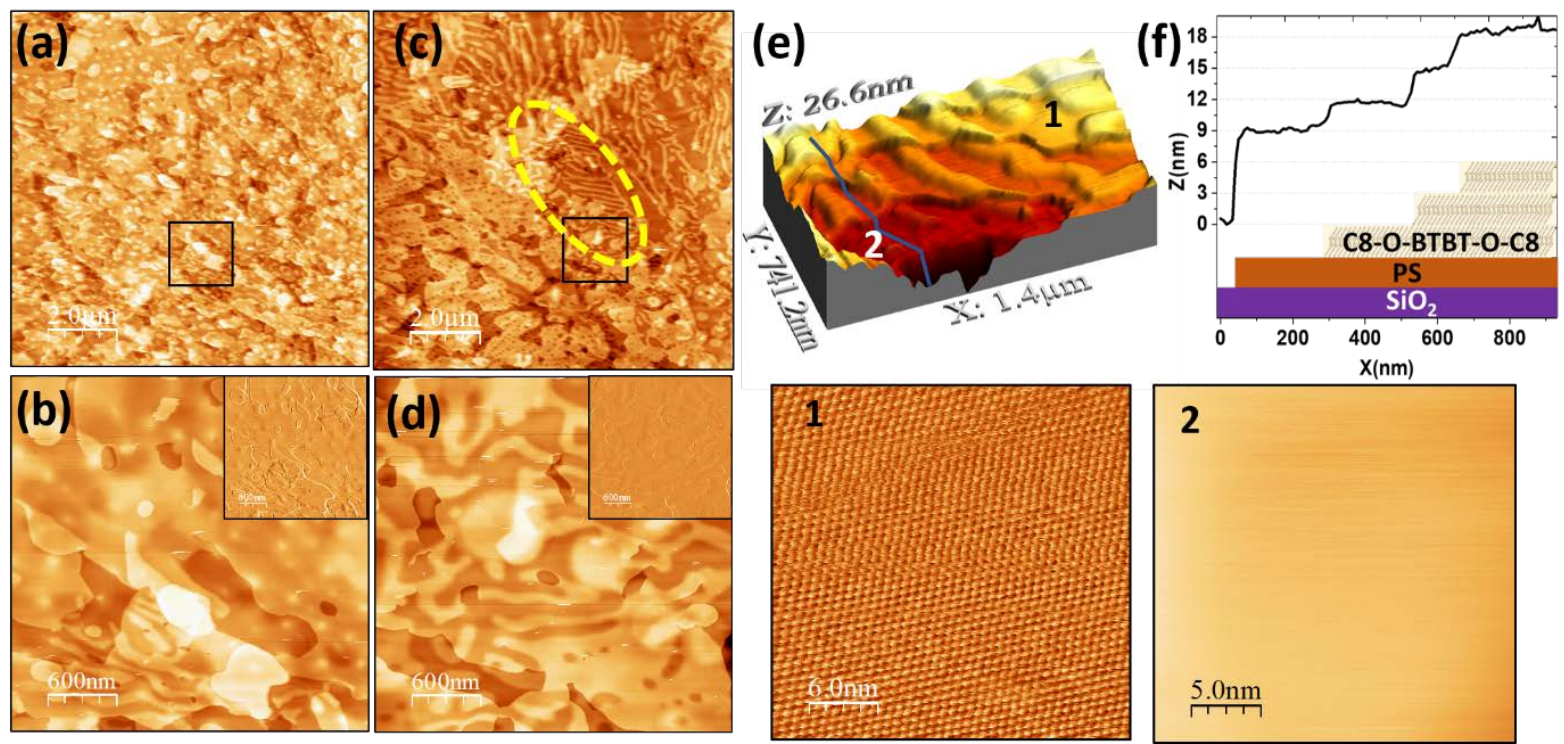

Figure 4. Topographic images for $\mathrm{C}_{8} \mathrm{O}-\mathrm{BTBT}-\mathrm{OC}_{8}: \mathrm{PS} 3 \mathrm{~K}(\mathrm{a}, \mathrm{b})$ and $\mathrm{C}_{8} \mathrm{O}-\mathrm{BTBT}-\mathrm{OC}$ :PS100K (c, d). The bottom images correspond to the areas marked by black squares in the large-scale images. Insets: respective frequency shift images. (e) 3D representation of a topographic image of $\mathrm{C}_{8} \mathrm{O}-\mathrm{BTBT}-\mathrm{OC}_{8}: \mathrm{PS} 100 \mathrm{~K}$ after the layer-by-layer peeling experiment detailed in the Supporting Information. (f) Topographic profile taken at the line marked in (e). Panels 1 and 2 at the bottom are representative high-resolution lateral force maps for top layers and bottom layers, as indicated in $(\mathrm{e})$. The measured periodicity $(\approx 5.0 \pm 0.5 \AA$ and $\approx 6.5 \pm 0.5 \AA)$ agrees within the error with the distances between molecules in the lattice as indicated in Figure S15c (d=4.9 $\AA$ and $\mathrm{a}=6 \AA \AA$ ).
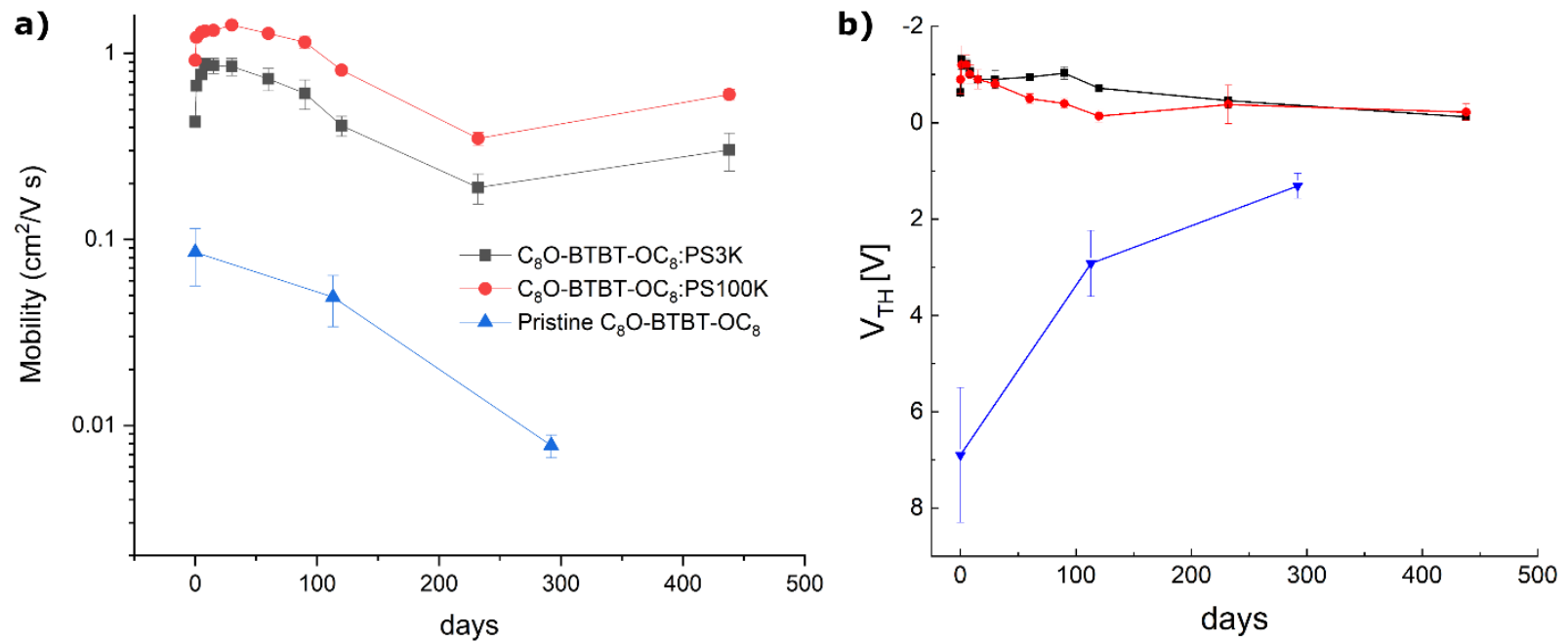

Figure 5. a) Mobility and b) threshold voltage evolution with time of devices based on pristine $\mathrm{C}_{8} \mathrm{O}-\mathrm{BTBT}-\mathrm{OC}_{8}$ (blue line), $\mathrm{C}_{8} \mathrm{O}-\mathrm{BTBT}-\mathrm{OC}_{8}: \mathrm{PS}_{3 \mathrm{~K}}$ (black line) and $\mathrm{C}_{8} \mathrm{O}-\mathrm{BTBT}-\mathrm{OC}_{8}: \mathrm{PS} 100 \mathrm{~K}$ (red line) deposited at $10 \mathrm{~mm} / \mathrm{s}$ and $105^{\circ} \mathrm{C}$. Notice that in the case of the pristine $\mathrm{C}_{8} \mathrm{O}-\mathrm{BTBT}$ $\mathrm{OC}_{8}$ OFETs, the devices stopped working after 300 days due to film dewetting. 
